\title{
The Palmar Cutaneous Branch of the Median Nerve: A Detailed Morphometric Study
}

\author{
Orane Richards ${ }^{1}$, Scott Border 1 , Claire Bolton ${ }^{2}$, Alexandra L. Webb ${ }^{1,3 *}$ \\ ${ }^{1}$ Centre for Learning Anatomical Sciences, Faculty of Medicine, University of Southampton, Southampton, UK \\ ${ }^{2}$ Orthopaedic Surgery Unit, The Canberra Hospital, Canberra, Australia \\ ${ }^{3}$ Medical School, Australian National University, Canberra, Australia \\ Email: ${ }^{*}$ alexandra.webb@anu.edu.au
}

Received 8 August 2014; revised 10 September 2014; accepted 7 October 2014

Copyright (C) 2014 by authors and Scientific Research Publishing Inc.

This work is licensed under the Creative Commons Attribution International License (CC BY).

http://creativecommons.org/licenses/by/4.0/

cc) (i) Open Access

\begin{abstract}
Damage to the palmar cutaneous branch of the median nerve (PCBMN) is a potential complication of surgery at the volar aspect of the wrist. The aim of this study was to determine the relationships of the PCBMN to both surface and bony landmarks using reliable methods. Ten pairs of forearms from cadavers aged 73 to 98 years were dissected. The PCBMN was identified and its course and relationships documented. The situation of the PCBMN was quantified relative to the distal wrist crease, bistyloid line, scaphoid tubercle, radial styloid process and flexor carpi radialis tendon. A PCBMN was identified on $90 \%$ of sides. The PCBMN arose from the radial aspect of the median nerve 52.4 (SD 31.0) $\mathrm{mm}$ from the bistyloid line and entered the transverse carpal ligament 10.9 (SD 9.5) mm proximal to the bistyloid line. At the level of the distal wrist crease the PCBMN was located 0.6 to $7.5 \mathrm{~mm}$ (mean 4.5; SD $1.9 \mathrm{~mm}$ ) from the ulnar aspect of the flexor carpi radialis tendon. The inter- and intra-observer reliability of the measurement methods ranged from ICC 0.96 to 1.00. Detailed morphometric data of the PCBMN relative to bony landmarks contribute to knowledge of the spatial relationships of the PCBMN to inform the precision of surgical incisions.
\end{abstract}

\section{Keywords}

Palmar Cutaneous Branch, Median Nerve, Morphometry, Gross Anatomy, Surgery

\section{Introduction}

The palmar cutaneous branch of the median nerve (PCBMN) has attracted much interest due to its vulnerability to direct trauma or accidental injury during a variety of surgical procedures around the volar forearm and wrist,

\footnotetext{
"Corresponding author.
} 
including carpal tunnel release, volar synovectomy, tendon transfer, ganglion resection and volar plate fixation of distal radius fractures [1]-[13]. Detailed knowledge of the disposition of neurovascular structures in relation to landmarks is important for the prevention of damage arising from surgical management [13] [14]. To date, the majority of cadaveric studies of the PCBMN have described and quantified the proximal-distal location of the PCBMN with reference to the distal wrist crease, which is frequently used as a surgical landmark [15]. Measurements from the distal wrist crease alone provide insufficient data to fully understand the proximal-distal and medial-lateral spatial relationships of the PCBMN. Furthermore, the variable location of the distal wrist crease may account for some of the variation in morphometric data reported for the PCBMN [3]. Moreover, the reliability of methods for measuring PCBMN morphometry has not been determined.

The purpose of this study was to quantify the proximal-distal and medial-lateral relationships of the PCBMN in relation to palpable bony landmarks, compared to the distal wrist crease, using statistically reliable measurement methods, and determine whether these were affected by sex and left or right sides.

\section{Materials and Methods}

Ten pairs of forearms, with hands attached, from six female and four male embalmed human cadavers aged 73 to 98 years (mean 89.4, SD 8.11) with no obvious signs of surgery, muscle wasting or pathology were dissected at the Centre for Learning Anatomical Sciences, University of Southampton. The study was approved by the institutional ethics committee.

Each forearm was dissected proximal to distal to first expose the median nerve and then the PCBMN. Once the PCBMN was identified the median nerve was exposed proximal to distal to ascertain the extent of the PCBMN and to avoid creating a false point of origin. Related neurovascular and musculoskeletal structures were dissected and a pin inserted into each bony landmark after they were clearly exposed by dissection. The morphology and relationships of the PCBMN were documented, including the incidence and pattern of anomalies. The following measurements were made using calibrated digital calipers (Mitutoyo, Tokyo, Japan) with the forearm in supination:

- Origin of the PCBMN (from the median nerve) to the bistyloid line, scaphoid tubercle and distal wrist crease (proximal to distal distance) (Figure 1).

- Distance from the point at which the PCBMN pierced the transverse carpal ligament to the bistyloid line and distal wrist crease (proximal to distal distance) and scaphoid tubercle (medial to lateral distance).

- Between the PCBMN (where it crossed the distal wrist crease) and the scaphoid tubercle, radial styloid process and the ulnar aspect of the FCR tendon (medial to lateral distance).

- Length of the PCBMN from its origin at the median nerve to the point at which it pierced/entered the transverse carpal ligament (proximal to distal distance). A piece of nylon thread was used to trace the true length of the PCBMN and the length of the thread was then measured using the calipers.

The most superficial aspect of the scaphoid tubercle was used for all measurements. Measurements were made three times using the blind side of the caliper, which was reset to zero after each measurement, and the mean calculated. All measurements were repeated within ten days and a second observer was trained to perform the measurements for the determination of intra-observer reliability and inter-observer reliability, respectively.

Data was presented as mean and standard deviation values with minimum and maximum values to indicate the extent of individual variation. Differences between left and right sides and male and female subjects were compared using paired and unpaired t-tests, respectively (significance level $\mathrm{p}=0.05$ ). Intra-observer reliability and inter-observer reliability were determined using intra-class correlation coefficients ICC $(2,1)$ and ICC $(3,1)$, respectively. All analyses were performed using SPSS version 17.0 (SPSS Inc., Chicago, Illinois, USA).

\section{Results}

A PCBMN was identified on 18 (90\%) of 20 sides (absent on two right sides, one male and one female). When present, the PCBMN arose from the radial aspect of the median nerve a mean of 55.15 (SD 28.69) mm proximal to the distal wrist crease, 76.04 (SD 31.89) mm proximal to the scaphoid tubercle and 52.38 (SD 31.55) mm proximal to the bistyloid line (Table 1, Figure 1). The PCBMN remained bound to the median nerve by connective tissue before it emerged obliquely and radially from the lateral aspect of the flexor digitorum superficialis muscle to lie a mean 4.46 (SD 1.91) mm, 22.82 (SD 14.06) $\mathrm{mm}$ and 29.07 (SD 6.57) mm medial to the ulnar aspect of the flexor carpi radialis (FCR) tendon, scaphoid tubercle and radial styloid process, respectively, at the 
Table 1. Palmar cutaneous branch of the median nerve (PCBMN) mean (SD) and [minimum - maximum] morphometric measures (mm) in the left and right forearms of male and female subjects.

\begin{tabular}{|c|c|c|c|c|c|c|}
\hline & \multicolumn{6}{|c|}{ Distance between the origin of the PCBMN to the: } \\
\hline & \multicolumn{2}{|c|}{ Bistyloid line } & \multicolumn{2}{|c|}{ Scaphoid tubercle } & \multicolumn{2}{|c|}{ Distal wrist crease } \\
\hline & Right & Left & Right & Left & Right & Left \\
\hline Female & $58.1(21.4)$ & $59.4(45.8)$ & $81.7(17.9)$ & $82.4(48.1)$ & $61.0(19.5)$ & $60.8(41.2)$ \\
\hline Male & $25.6(8.9)$ & $54.7(24.5)$ & $50.1(7.5)$ & $78.8(24.9)$ & $59.7(17.8)$ & $47.9(8.5)$ \\
\hline \multirow[t]{4}{*}{ All } & $\begin{array}{c}45.9(23.8) \\
{[15.4-93.9]}\end{array}$ & $\begin{array}{c}57.6(37.1) \\
{[16.0-137.0]}\end{array}$ & $\begin{array}{c}69.9(21.6) \\
{[41.7-112.9]}\end{array}$ & $\begin{array}{c}81.0(38.7) \\
{[31.6-164.0]}\end{array}$ & $\begin{array}{c}48.7(23.8) \\
{[12.5-89.9]}\end{array}$ & $\begin{array}{c}60.3(32.4) \\
{[25.6-130.9]}\end{array}$ \\
\hline & \multicolumn{6}{|c|}{ Distance from site of PCBMN piercing the transverse carpal ligament to the: } \\
\hline & \multicolumn{2}{|c|}{ Bistyloid line } & \multicolumn{2}{|c|}{ Scaphoid tubercle } & \multicolumn{2}{|c|}{ Distal wrist crease } \\
\hline & Right & Left & Right & Left & Right & Left \\
\hline Female & $14.2(16.3)$ & $9.9(6.6)$ & $27.6(21.8)$ & $23.5(13.9)$ & $14.8(14.5)$ & $10.3(4.6)$ \\
\hline Male & $13.2(4.7)$ & $6.5(4.4)$ & $16.14(6.8)$ & $20.4(9.4)$ & $8.2(9.7)$ & $8.0(2.3)$ \\
\hline \multirow[t]{4}{*}{ All } & $\begin{array}{l}13.8(12.6) \\
{[1.0-40.8]}\end{array}$ & $\begin{array}{c}8.6(5.8) \\
{[1.3-18.6]}\end{array}$ & $\begin{array}{l}23.3(17.9) \\
{[8.0-64.1]}\end{array}$ & $\begin{array}{l}22.3(11.8) \\
{[3.8-42.1]}\end{array}$ & $\begin{array}{l}12.3(12.6) \\
{[1.5-38.8]}\end{array}$ & $\begin{array}{c}9.4(3.9) \\
{[5.1-18.5]}\end{array}$ \\
\hline & \multicolumn{6}{|c|}{ Distance between PCBMN, at the level of the distal wrist crease, and the: } \\
\hline & \multicolumn{2}{|c|}{ Scaphoid tubercle } & \multicolumn{2}{|c|}{ Radial styloid process } & \multicolumn{2}{|c|}{ FCR tendon* } \\
\hline & Right & Left & Right & Left & Right & Left \\
\hline Female & $30.1(21.3)$ & $21.8(12.1)$ & 31.9 (11.4) & $29.2(4.1)$ & $4.3(2.4)$ & $4.5(0.9)$ \\
\hline Male & $16.1(6.6)$ & $20.3(10.0)$ & 25.9 (1.7) & $27.8(4.0)$ & $6.3(1.0)$ & $3.3(2.4)$ \\
\hline All & $\begin{array}{l}24.8(18.0) \\
{[8.4-65.1]}\end{array}$ & $\begin{array}{l}21.2(10.7) \\
{[3.6-35.6]}\end{array}$ & $\begin{array}{c}29.6(9.2) \\
{[22.8-51.3]}\end{array}$ & $\begin{array}{c}28.6(3.4) \\
{[24.1-34.0]}\end{array}$ & $\begin{array}{c}5.0(2.2) \\
{[2.2-7.5]}\end{array}$ & $\begin{array}{c}4.0(1.7) \\
{[0.6-5.6]}\end{array}$ \\
\hline
\end{tabular}

*Ulnar aspect.

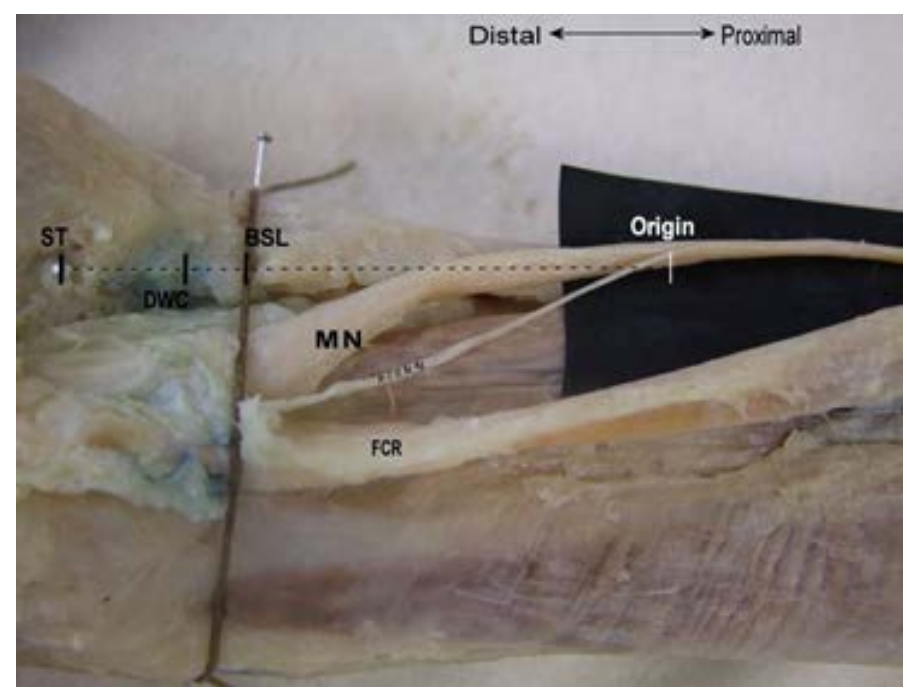

Figure 1. Palmar cutaneous branch (PCBMN), of the right forearm, at its origin from the median nerve $(\mathrm{MN})$. Measurements were made from the PCBMN origin to the bistyloid line (BSL), distal wrist crease (DWC) and scaphoid tubercle (ST). 
level of the distal wrist crease (Table 1). The nerve then entered its own tunnel within the transverse carpal ligament, however, in seven specimens the PCBMN gave off a small branch to the fascia of the transverse carpal ligament before doing so. The point at which the nerve entered the transverse carpal ligament was a mean of 10.88 (SD 9.48) $\mathrm{mm}$ and 10.69 (SD 8.68) $\mathrm{mm}$ distal to the bistyloid line and distal wrist crease, respectively, and a mean of 22.73 (SD 14.35) mm medial to the scaphoid tubercle (Table 1). The average length of the PCBMN was 57.40 (SD 29.15) mm (females right 54.1 (SD 5.6) and left 67.1 (SD 45.3); males right 39.1 (SD 5.5) and left 60.7 (SD 27.9)).

With the exception of distance between the PCBMN and the ulnar aspect of the FCR tendon, all measurements of the PCBMN were generally greater in female compared to male subjects (Table 1). Within individuals, the morphometry of the PCBMN often varied between left and right sides (Table 1). No statistical differences were identified between sexes or sides. Intra-observer and inter-observer reliability were excellent for all measurements and ranged from ICC 0.99 to 1.00 and 0.96 to 1.00 , respectively.

\section{Discussion}

In this study, the location of the PCBMN relative to palpable osseous landmarks and the distal wrist crease was investigated to determine the proximal-distal and medial-lateral relationships of the PCBMN at the wrist. We found greater consistency between measures of the PCBMN to the bistyloid line [3] [16] compared to the distal wrist crease [1] [3] [4] [6] [9] [17]-[20]. The PCBMN arose from the radial aspect of the median nerve a mean of 52.4 (SD 31.0) $\mathrm{mm}$ and entered the transverse carpal ligament a mean of 10.9 (SD 9.5) mm proximal to the bistyloid line, respectively. At the wrist, the PCBMN was located 0.6 to $7.5 \mathrm{~mm}$ (mean 4.5; SD $1.9 \mathrm{~mm}$ ) medial to the ulnar aspect of the flexor carpi radialis tendon.

Topographical markers and detailed knowledge of the disposition of the PCBMN in relation to landmarks are important in ensuring the preciseness of surgical incisions to prevent iatrogenic injury and ensure a good outcome [14] [15]. Whilst superficial skin markers such as the distal wrist crease are typically used for the planning of surgical incisions, there has been some concern regarding the variability of skin creases [3]. Bony landmarks may be relevant to the prevention of PCBMN damage arising from surgical interventions at the volar aspect of the wrist, when structures such as the FCR tendon are difficult to palpate or the PCBMN is difficult to visualise due to pronounced soft tissue swelling or trauma [7]. Previous studies have focused on proximal-distal measures between the PCBMN and the distal wrist crease, overlooking both the relationships to bony landmarks and the medial-lateral disposition of the PCBMN. Thus there is insufficient data to understand the three-dimensional anatomy and spatial relationships of the PCBMN at the wrist. Anatomical studies such as this one are needed since MR studies currently do not enable sufficient visualisation of the PCBMN [13] [21]. This paper contributes to the creation of an accurate representation of the anatomy of the PCBMN on the volar aspect of the forearm and wrist. Dissection based studies are very often limited to a sample of elderly embalmed cadavers, and so it is suggested that future studies could be conducted using ultrasound to examine the location of the PCBMN in living subjects. This has the advantage of exploring potential age related differences along with surface and bony landmarks to help extend our understanding of the precise anatomy of this nerve relevant to the planning of safe surgical incisions [12] [15].

The range of values measured between the PCBMN and the distal wrist crease and bistyloid line were similar to previous measurements [3] [6] [17]. The location of the PCBMN relative to the bistyloid line was, on average, within $15 \mathrm{~mm}$ of measures made from the distal wrist crease. This finding is in agreement with Chaynes et al. [3] who determined that the distal wrist crease was located between 0 and $11.2 \mathrm{~mm}$ distal to the bistyloid line. The mean length of the PCBMN in the present study was similar to the $52.4 \mathrm{~mm}$ and $54.6 \mathrm{~mm}$ recorded by Bezerra et al. [17] and Chaynes et al. [3], respectively, but less than the $12.9 \mathrm{~cm}$ reported by Hobbs et al. [7]. The distance between the PCBMN and radial styloid process and FCR tendon were comparable with measures made by Kwiatkowska et al. [15] from the radial side of the hand and by McCann et al. [13] at the distal physeal scar of the radius.

The PCBMN was absent in two of the twenty specimens dissected, which is consistent with the findings of Born et al. [22] and Carare et al. [23]. The PCBMN has previously been described as consistently present [3] [4] [6] [7] [9] [16] [20]. It is possible that the PCBMN had a higher origin in limbs in which only the forearm was available for study and is a limitation of studies in which the cadaveric specimens are primarily used for teaching purposes. Our observations confirmed that the PCBMN entered its own fibrous tunnel in the transverse car- 
pal ligament, but in seven specimens it gave off a small branch to the deep fascia of the transverse carpal ligament [1].

The wide range of measures in this and previous studies may reflect the individual diversity of subjects. In future studies, it may be beneficial to measure the dimensions of each individual's forearm, wrist and hand in order to normalise the measurements of PCBMN morphometry. This would facilitate comparisons between studies and may account for potential differences. It is of course possible that some variations may be the result of methodological differences. Bony landmarks were chosen in the present study as it was felt these would be more reproducible than measures from the distal wrist crease as used in previous studies. The intra- and inter-observer reliability was found to be equal for measurements made from the bistyloid line, scaphoid tubercle and distal wrist crease. The reliability of measurement methods used in previous studies has not been documented. The authors would recommend that any further anatomical investigations of this nature adopt reliability measures as a way of ensuring more meaningful comparisons of the data in the future.

\section{Conclusion}

The detailed knowledge of the morphometry of the PCBMN in relation to bony landmarks enhances understanding of the three-dimensional anatomy and relationships of the PCBMN which may assist in surgical applications in the region of the volar aspect of the forearm and wrist.

\section{Acknowledgements}

We wish to thank Mr. Fraser Chisholm for his assistance with the reliability study and Ms. Elizabeth O’Sullivan for advice during the dissection process.

\section{References}

[1] Al-Qattan, M.M. (1997) Anatomical Classification of Sites of Compression of the Palmar Cutaneous Branch of the Median Nerve. The Journal of Hand Surgery: British \& European Volume, 22, 48-49. http://dx.doi.org/10.1016/S0266-7681(97)80014-9

[2] Bergland, L.M. and Messer, T.M. (2009) Complications of Volar Plate Fixation for Managing Distal Radius Fractures. Journal of the American Academy of Orthopaedic Surgeons, 17, 369-377.

[3] Chaynes, P., Becue, J., Vaysse, P. and Laude M. (2004) Relationships of the Palmar Cutaneous Branch of the Median Nerve: A Morphometric Study. Surgical and Radiologic Anatomy, 26, 275-280. http://dx.doi.org/10.1007/s00276-004-0226-2

[4] Cheung, J.W., Shyu, J.F., Teng, C.C., Chen, T.H., Su, C.H., Shyr, Y.M., Wang, J.J., Wu, C.W., Lui, W.Y. and Liu, J.C. (2004) The Anatomical Variations of the Palmar Cutaneous Branch of the Median Nerve in Chinese Adults. Journal of the Chinese Medical Association, 67, 27-31.

[5] Das, S.K. and Brown H.G. (1976) In Search of Complications in Carpal Tunnel Decompression. Hand, 8, $243-249$. http://dx.doi.org/10.1016/0072-968X(76)90009-7

[6] DaSilva, M.F., Moore, D.C., Weiss, A.P.C., Akelman, E. and Sikirica, M. (1996) Anatomy of the Palmar Cutaneous Branch of the Median Nerve: Clinical Significance. The Journal of Hand Surgery: American Volume, 21, 639-643. http://dx.doi.org/10.1016/S0363-5023(96)80018-9

[7] Hobbs, R.A., Magnussen, P.A. and Tonkin, M.A. (1990) Palmar Cutaneous Branch of the Median Nerve. The Journal of Hand Surgery: American Volume, 15, 38-43. http://dx.doi.org/10.1016/S0363-5023(09)91103-0

[8] Louis, D.S., Greene, T.L. and Noellert, R.C. (1985) Complications of Carpal Tunnel Surgery. Journal of Neurosurgery, 62, 352-356. http://dx.doi.org/10.3171/jns.1985.62.3.0352

[9] Matloub, H.S., Yan, J.G., Van Der Molen, A.B.M., Zhang, L.L. and Sanger, J.R. (1998) The Detailed Anatomy of the Palmar Cutaneous Nerves and Its Clinical Implications. The Journal of Hand Surgery: British \& European Volume, 23, 373-379. http://dx.doi.org/10.1016/S0266-7681(98)80061-2

[10] Naff, N., Dellon, A.L. and Mackinnon, S.E. (1993) The Anatomical Course of the Palmar Cutaneous Branch of the Median Nerve including a Description of Its Own Unique Tunnel. Journal of Hand Surgery, 18, 316-317. http://dx.doi.org/10.1016/0266-7681(93)90050-P

[11] Taleisnik, J. (1973) The Palmar Cutaneous Branch of the Median Nerve and the Approach to the Carpal Tunnel: An Anatomical Study. Journal of Bone and Joint Surgery: American Volume, 55, 1212-1217.

[12] Tagliafico, A., Pugliese, F., Bianchi, S., Bodner, G., Padua, L., Rubino, M. and Martinoli, C. (2008) High-Resolution 
Sonography of the Palmar Cutaneous Branch of the Median Nerve. American Journal of Roentgenology, 191, 107-114. http://dx.doi.org/10.2214/AJR.07.3383

[13] McCann, P.A., Clarke, D., Amirfeyz, R. and Bhatia, R. (2012) The Cadaveric Anatomy of the Distal Radius: Implications for the Use of Volar Plates. Annals of the Royal College of Surgeons of England, 94, 116-120. http://dx.doi.org/10.1308/003588412X13171221501186

[14] Olave, E., Del Sol, M., Gabriellp, C., Mandiola, E. and Rodrigues, C.F. (2001) Biometric Study of the Relationships between Palmar Neurovascular Structures, the Flexor Retinaculum and the Distal Wrist Crease. Journal of Anatomy, 198, 737-741. http://dx.doi.org/10.1046/j.1469-7580.2001.19860737.x

[15] Kwiatkowska, M., Jakutowicz, T., Ciszek, B. and Czubak, J. (2014) Can Palmar Creases Serve as Landmarks for the Deeper Neuro-Vascular Structures? Surgical and Radiologic Anatomy, 36, 495-501. http://dx.doi.org/10.1007/s00276-013-1211-4

[16] Bonnel, F., Mailhe, P., Allieu, Y. and Rabischong, P. (1980) Bases Anatomiques de la Chirurgie Fasciculaire du Nerf Median au Poignet. Annales de Chirurgie, 34, 707-710.

[17] Bezerra, A.J., Carvalho, V.C. and Nucci, A. (1986) An Anatomical Study of the Palmar Cutaneous Branch of the Median Nerve. Surgical and Radiologic Anatomy, 8, 183-188. http://dx.doi.org/10.1007/BF02427847

[18] Dowdy, P., Richards, R. and McFarlane, R. (1994) The Palmar Cutaneous Branch of the Median Nerve and the Palmaris Longus Tendon. A Cadaveric Study. Journal of Hand Surgery, 19A, 199-202. http://dx.doi.org/10.1016/0363-5023(94)90005-1

[19] Alizadeh, K., Lahiji, F. and Phalsaphy, M. (2006) Safety of Carpal Tunnel Release with a Short Incision. A Cadaver Study. Acta Orthopaedica Belgica, 72, 415-419.

[20] Ozcanli, H., Coskun, N.K., Cengiz, M., Oguz, N. and Sindel, M. (2010) Definition of a Safe-Zone in Open Carpal Tunnel Surgery: A Cadaver Study. Surgical and Radiologic Anatomy, 32, 203-206. http://dx.doi.org/10.1007/s00276-009-0498-7

[21] McCann, P.A., Amirfeyz, R., Wakeley, C. and Bhatia, R. (2010) The Volar Anatomy of the Distal Radius-An MRI Study of the FCR Approach. Injury, 4, 1012-1014. http://dx.doi.org/10.1016/j.injury.2010.02.031

[22] Born, T. and Mahoney, J. (1995) Cutaneous Distribution of the Ulnar Nerve in the Palm: Does It Cross the Incision Used in Carpal Tunnel Release? Annals of Plastic Surgery, 35, 23-25. http://dx.doi.org/10.1097/00000637-199507000-00005

[23] Carare, et al. (2006) Personal Communication. 
Scientific Research Publishing (SCIRP) is one of the largest Open Access journal publishers. It is currently publishing more than 200 open access, online, peer-reviewed journals covering a wide range of academic disciplines. SCIRP serves the worldwide academic communities and contributes to the progress and application of science with its publication.

Other selected journals from SCIRP are listed as below. Submit your manuscript to us via either submit@scirp.org or Online Submission Portal.
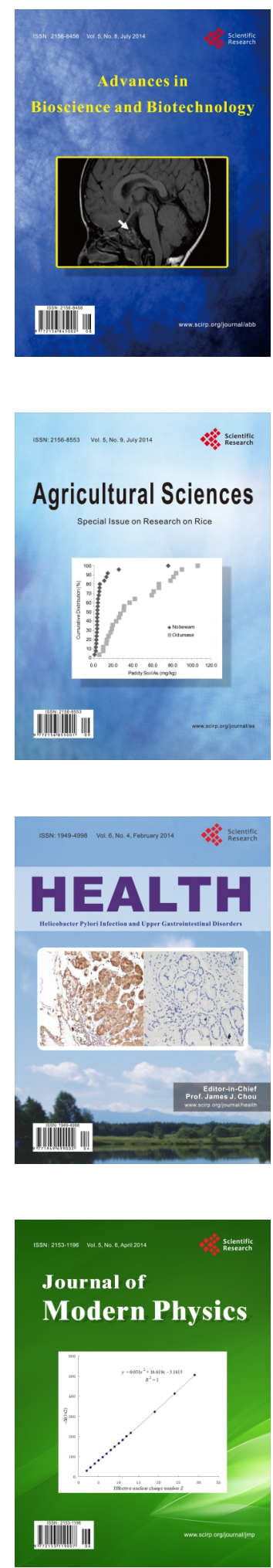
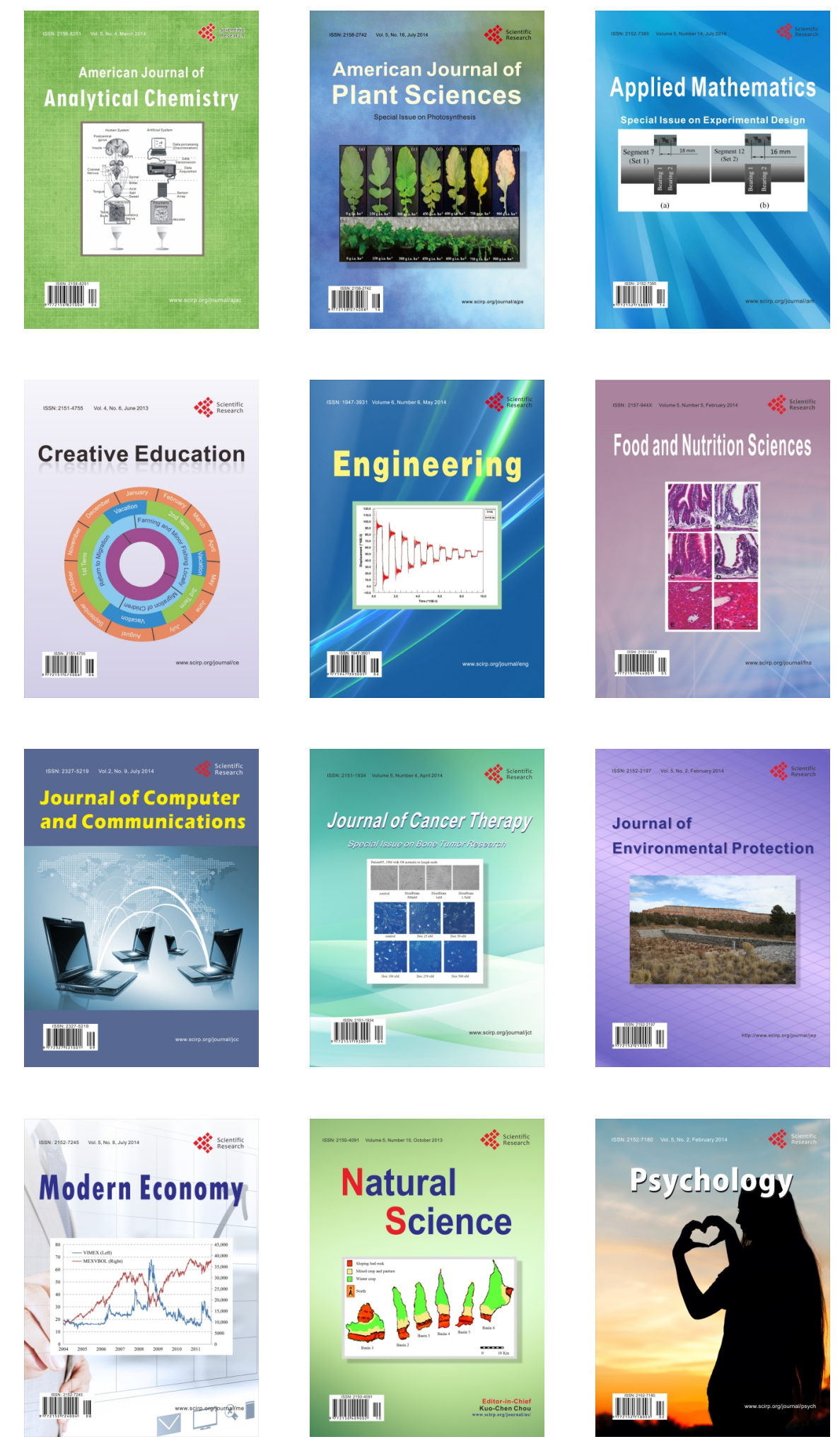